\title{
NUCLEAR QUADRUPOLE RESONANCE INVESTIGATION OF PHASE TRANSITION IN TETRAMETHYLAMMONIUM CADMIUM TRIBROMIDE
}

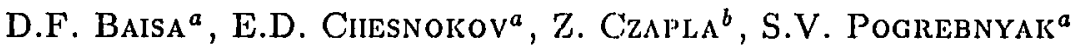 \\ AND I.G. VERTEGEL ${ }^{a}$ \\ ${ }^{a}$ Institute of Physics, Ukrainian Acad. Sci. \\ Prospekt Nauki 46, 252028 Kiev, Ukraine \\ ${ }^{b}$ Institute of Experimental Pliysics, University of Wrocław \\ Pl. Maxa Borna 9, 50-204 Wroclaw, Poland
}

(Received Oclober 6, 1992; revised version May 18, 1993)

\begin{abstract}
The critical temperature dependence of the nuclear quadrupole resonance ${ }^{79} \mathrm{Br}$ frequencies at the phase transition $\left(T_{c}=163 \mathrm{~K}\right)$ was found in $\left(\mathrm{CH}_{3}\right)_{4} \mathrm{NCdBr}_{3}$. It was concluded that bromine nuclei take part in the phase transition not directly but via the interaction with the crystal polarization.
\end{abstract}

PACS numbers: $64.70 . \mathrm{Kb}$

Tetramethylammonium cadmium tribromide, $\left(\mathrm{CII}_{3}\right)_{4} \mathrm{NCdBr}_{3}$ (TMCB), belongs to the compounds of the type $\left(\mathrm{CII}_{3}\right)_{4} \mathrm{NMX}_{3}(\mathrm{M}=\mathrm{Mn}, \mathrm{Ni}, \mathrm{Cd} ; \mathrm{X}=\mathrm{Cl}, \mathrm{Br}, \mathrm{I})$, which also includes such widely investigated crystals as $\left(\mathrm{CII}_{3}\right)_{4} \mathrm{NMnCl}_{3}$ (TMMC) and $\left(\mathrm{CH}_{3}\right)_{4} \mathrm{NCdCl}_{3}$ (TMCC). The peculiarity of their structure is the existence of infinite chains $\left(\ldots \mathrm{M}-\mathrm{X}_{3}-\mathrm{M}-\mathrm{X}_{3} \ldots\right)$ separated by tetramethylammonium ions. These ions are orientationally disordered at room temperature [1, 2]. At low temperatures these compounds are characterized by the structural phase transition of the order-disorder type, accompanied by ordering of the tetramethylammonium ions $[3,4]$.

At room temperature the TMCB crystal is isomorplous to the above-mentioned compounds and the first-order structural phase transition occurs at the temperature $T=163 \mathrm{~K}$ [5]. IIowever, the NMR investigations did not reveal any anomalies in the temperature dependence of the proton spin-lattice relaxation time at $163 \mathrm{~K}[6]$. This phenomenon may be explained by the fact that in this temperature range the relaxation mechanism is due to fast reorientation of the $\mathrm{CH}_{3}$-groups which masks slower motions connected with the change of structure at the phase transition. 
The method of nuclear quadrupole resonance (NQR) was used to elucidate the role of bromine nuclei in the mechanism of the phase transition and to investigate the lattice dynamics in the phase transition temperature region.

The ${ }^{79} \mathrm{Br}$ NQR investigation of $\mathrm{TMCB}$ has shown that at $T=300 \mathrm{~K}$ the spectrum contains a single line of frequency $\nu=55.200 \mathrm{MHz}$. At $T=77 \mathrm{~K}$ the triplet was found with the following frequencies: $\nu_{1}=55.750 \mathrm{MII}, \nu_{2}=56.770 \mathrm{MIz}$ and $\nu_{3}=60.180 \mathrm{MHz}$. With a temperature increase from $77 \mathrm{~K}$ to $163 \mathrm{~K}$ the intensity of these three lines decreases and at $T_{\mathrm{c}}=163 \mathrm{~K}$ the spectrum multiplicity changes abruptly. The temperature dependence of the ${ }^{79} \mathrm{Br}$ NQR frequencies in polycrystalline TMCB is shown in Fig. 1. No hysteresis phenomena have been found in

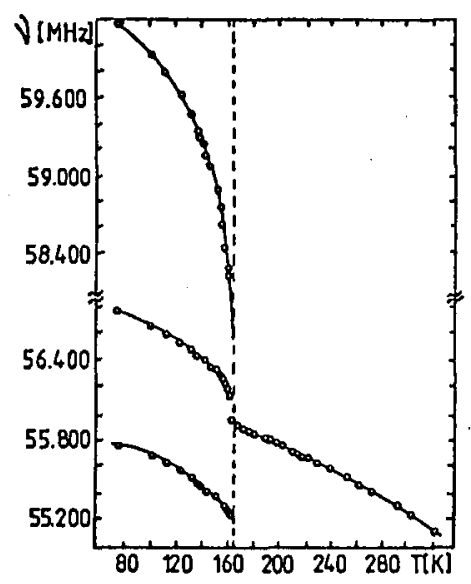

Fig. 1. The temperature dependence of the NQR ${ }^{79} \mathrm{Br}$ frequencies in TMCB.

the phase transition region within the experimental accuracy of $\pm 0.5 \mathrm{~K}$. This fact does not contradict the data of dielectric measurements where the hysteresis was equal to $0.2 \mathrm{~K} \mathrm{[5].} \mathrm{The} \mathrm{authors} \mathrm{of} \mathrm{this} \mathrm{article} \mathrm{classify} \mathrm{the} \mathrm{pliase} \mathrm{transition} \mathrm{as} \mathrm{ferro-}$ electric of the order-disorder type with the first-order transition features. A similar dependence of NQR frequencies for the ${ }^{79} \mathrm{Br}$ was obtained by Nakamura [7].

The temperature bevaviour of the three NQR lines is critical as it can be seen in Fig. 1. We tried to single out the critical contribution to NQR parameters. It is supposed that the rearrangement of several atoms in an elementary unit cell in the order-disorder phase transition occurs [8] and the degrees of freedom of other atoms form a thermostat. In this case noncritical degrees of freedom of oscillations cause the usual temperature dependence of NQR frequencies [9] which in a high temperature region is assumed to be of the form

$$
\nu(T)=a\left(1+b T+c T^{-1}\right)
$$

where $a=\nu_{0}$ is the NQR frequency at $T=0 \mathrm{~K}$ without averaging on zero point vibration,

$$
b=-\frac{3 K}{8 \pi^{2}} \sum_{i} \frac{n_{i}}{I_{i} \nu_{i}^{2}}, \quad c=-\frac{h^{2}}{32 \pi^{2} K} \sum_{i} \frac{1}{I_{i}}
$$


$\nu_{i}$ is the frequency of torsional vibrations of the resonance nucleus, $I_{i}$ is the inertia moment of the torsional vibrations, $n_{i}$ is the degeneration degree of the given oscillation. After such a treatment of the temperature dependence of the NQR frequencies at $T>T_{c}$, one gets the following values for $a, b, c$ parameters: $a=$ $57.353 \pm 0.005 \mathrm{MIIz}, b=(-1.114 \pm 0.008) 10^{-4} \mathrm{~K}^{-1}, c=(-1.079 \pm 0.01) \mathrm{K}$. It is known that the NQR frequency $\nu_{0}$ is determined by the value of the intracrystalline electrical field gradient at the bromine nuclei. If we assume that the bromine nuclei do not take part directly in the critical dynamics and other atoms groups (for example $\mathrm{N}\left(\mathrm{CHI}_{3}\right)_{4}$ ) undergo ordering at the phase transition in this crystal, the critical behaviour of NQR spectrum parameters at the phase transition is determined by the inluence of macroscopic changes of the crystal on the local electric field gradient tensor of the bromine nuclei. Taking into account that the ferroelectric phase is present below the phase transition temperature [5] it can be shown that the parameters of the tensor of electric field gradient at the bromine nuclei has the following form:

$$
q_{i j}=q_{i j}^{0}\left(1+\sum_{k} R_{i j k} P_{k}\right),
$$

where $P_{k}$ are the polarization vector components in respect to the crystallographic axes; $R_{i j k}$ is a third rank tensor. Such a form considers the fact that the symmetry of the surrounding of the bromine nuclei lias no inversion centre. That is why it is possible to confine to the linear expansion coefficient in respect to the polarization. Then, performing the standard procedure of obtaining the NQR frequencies [10] and taking into account the usual temperature dependence of polarization in case of the phase transition of the order-disorder type the following relation between the frequency for the rigid lattice and the temperature can be obtained:

$$
\nu_{i}(P)=d_{i}+f_{i}\left(T_{\mathrm{c}}-T\right)^{1 / 2}
$$

where $i=1,2,3$ are the numbers of different NQR spectrum lines below $T_{\mathrm{c}}$. Thus, the temperature dependence of the resonance frequencies below $T_{\mathrm{c}}$ is of the following form:

$$
\nu_{i}(T)=\left[d_{i}+f_{i}\left(T_{\mathrm{c}}-T\right)^{1 / 2}\right]\left(1+b T+c T^{-1}\right) .
$$

The experimental dependence of the NQR frequencies ${ }^{79} \mathrm{Br}$ can be satisfactorily described by the theoretical dependence for $d_{i}$ and $f_{i}$ shown in Table.

\section{TABLE}

The coefficients $d_{i}$ and $f_{i}$ used in Eqs. (3) and (4).

\begin{tabular}{l|r|r|r}
\hline \hline The spectrum line number & \multicolumn{1}{c|}{1} & \multicolumn{1}{c|}{2} & \multicolumn{1}{c}{3} \\
\hline$d[\mathrm{MIIz}]$ & 59.55 & 57.58 & 56.61 \\
$f\left[\mathrm{MHz} \mathrm{K}^{-1 / 2}\right]$ & 0.22 & 0.05 & 0.04
\end{tabular}

The calculated constants $d_{i}$ and $f_{i}$ lave different values for the three lines. That is apparently connected with the different direction of the crystal polarization in respect to main axes of the tensor of electric lield gradient at the bromine nuclei. 
Thus, the obtained results allow one to conclude that the bromine nuclei in this crystal do not take part in the phase transition directly and their critical behaviour is caused by the interaction with the crystal polarization. The crystal undergoes the phase transition of the order-disorder type at which inertia moments and torsional vibrations of the bromine nuclei do not cliange.

\section{References}

[1] B. Morosin, E.J. Graeber, Acta Crystallogr. 23, 766 (1967).

[2] P.S. Peerey, B. Morosin, G. Samara, Phys. Rev. B 8, 3378 (1973).

[3] P.S. Peerey, B. Morosin, Phys. Lett. A 36, 409 (1971).

[4] M.T. Hutchings, G.S. Pawley, W.G. Stirling, J. Phys. C 16, 115 (1983).

[5] P. Vanek, M. Havrankova, F. Smutny, B. Brezina, Ferroclectrics 100, 51 (1990).

[6] K. Venu, S.S. Sastry, J. Ramakrishna, J. Phys. C 20, 1519 (1987).

[7] S. Ishimaru, N. Nakamura, J. Phys. Soc. Jpn. 61, 1787 (1992).

[8] R. Blinc, B. Zeks, Soft Modes in Ferroelectrics and Antiferroelectrics, North-Ifolland Publ. Co., Amsterdam, Oxford 1974.

[9] H. Bayer, Z. Phys. 130, 227 (1951).

[10] T.P. Das, E.L. Hahn, Nuclear Quadrupole Resonance Spectroscopy, in series Solid State Phys., Suppl. 1, Academic Press, New York 1958. 